\title{
Comparison of Lower Extremity Muscle Activity in Sliding Lunges versus Standard Lunges
}

Daniel Drill, Adam Fong, Victoria Voorhees, Rumit Singh Kakar, Deborah L. King. Ithaca College, Ithaca, NY

Lunges are a functional exercise used in lower body neuromuscular training programs. Lunges are typically performed on the floor stepping forward, sideways, or backwards, but can be performed on a slide board or with foot sliders. Sliding allows partial weight bearing and may improve stability. Differences in neuromuscular challenges, specifically hip and knee muscle activity, between sliding and standard lunges are unknown. PURPOSE: To compare hip and knee muscle activity between sliding and standard reverse lunges (RL) and side lunges (SL). METHODS: Sixteen healthy active subjects performed 3 types of RLs and SLs: slide board (SB), foot sliders (FS), and standard lunges (ST). After skin preparation, surface electrodes were placed over the muscle bellies of the gluteus maximus (GMX), ipsi- and contra-lateral gluteus medii (iGM, cGM), vastus lateralis (VL), and vastus medialis (VM). Muscle activity of the weight bearing leg was measured at $1000 \mathrm{~Hz}$ with a wireless EMG system. Lunges were performed at a standardized tempo (44 bpm) and subject specific standardized length. Maximum voluntary isometric contractions (MVIC) were used to normalize peak EMG (pEMG) and average EMG (aEMG) to percent MVIC for 4 lunges of each subject all conditions. One-way repeated measures ANOVAs $(\alpha=0.05)$ were used to determine differences between the 3 lunge types for SL and RL. RESULTS: RL cGM aEMG was greater for ST (22 $\pm 21 \%)$ versus FS (16 $\pm 17 \%), \mathrm{p}=.012$. RL VM aEMG was lower for SB $(30 \pm 12 \%)$ versus FS $(34 \pm 14 \%), \mathrm{p}$ $=.016$. SL VL aEMG was greater for ST $(230 \pm 150 \%)$ versus SB $(198 \pm 121 \%), p=.049$. SL GMX aEMG was greater for ST $(192 \pm 137 \%)$ versus SB $(159 \pm 123 \%)$ and FS $(162 \pm 103 \%)$, p $=.001$ and .035 . SL VM aEMG was greater for ST $(135 \pm 14 \%)$ versus SB $(26 \pm 11 \%)$ and FS $(25 \pm 9 \%), \mathrm{p}=.009$ and .004 . SL iGM pEMG was greater for ST $(79 \pm 44 \%)$ versus SB $(52 \pm$ $32 \%)$ and FS $(50 \pm 37 \%), p=.019$ and .003 . SL cGM pEMG was greater for ST $(79 \pm 41 \%)$ versus $\mathrm{SB}(59 \pm 34 \%), \mathrm{p}=.027$. SL VM pEMG was greater for ST $(92 \pm 46 \%)$ versus SB $(63 \pm$ $28 \%)$ and FS $(60 \pm 18 \%), p=.031$ and .01 . CONCLUSION: Hip and knee muscle activity is similar for RL with minor differences in cGM and VM based on lunge type. Standard SL had consistently greater peak and average muscle activity compared to sliding lunges. Sliding SL may require different neural control which could be important in rehabilitation settings. 